Full-Length Article

\title{
All Roads Lead To Where I Stand: A Veteran Case Review Kristen Stewart ${ }^{1}$ \\ ${ }^{1}$ VA - Hudson Valley Healthcare System, United States of America
}

\begin{abstract}
The purpose of this article is to illustrate individualized clinical practice in music therapy as part of treatment programming for veterans with PTSD. Multidimensional aspects of treatment are referenced through an integrative and multi-modal approach to highlight the coordination of processes in meeting veteran needs and goals. The incorporation of Somatic Experiencing ${ }^{\circledR}$ techniques will also be highlighted as a means of promoting stabilization and developing resiliency by increasing awareness of sensate experience to help resolve fixated physiologic states due to traumatic stress. Current trauma models will be reviewed to help explore additional needs for growth in music therapy practice in the treatment of veterans coping with trauma.
\end{abstract}

Keywords: trauma, post-traumatic stress disorder, integral psychology, felt sense, Somatic Experiencing ${ }^{\circledR}$

multilingual abstract $\mid$ mmd.iammonline.com

\section{Introduction}

Much of our history as a music therapy profession is rooted in the use of music and music-based services provided for veterans coping from physical and emotional traumas as a result of WWI and WWII. Primary goals during this time have been identified as boosting morale, supporting social readjustment and as occupational therapy [1]. Objectives in education and physical conditioning were added soon thereafter [2]. While the practice of music therapy has grown to address needs across the span of human experience since these foundational times, "the seeds planted early on in the military, however, have not mirrored the rate of growth and development of the profession as a whole." [3] Empiricalbased music therapy research focusing on military and veteran populations coping with trauma is similarly limited, with three noted studies [4].

Despite these limitations, an interest and demand for complementary, alternative and integrative approaches to treatment for veterans and military personnel is on the rise $[5,6]$. When coupled with statistics regarding the prevalence of Post-Traumatic Stress Disorder (PTSD) among veterans, it becomes clear that in addition to a desire for the types of

\section{PRODUCTION NOTES: Address correspondence to:}

Kirsten Stewart, E-mail: pjkmstewart@optonline.net | COI statement: The authors declared that no financial support was given for the writing of this article. The authors have no conflict of interest to declare.

Copyright () 2018 All rights reserved.

International Association for Music \& Medicine (IAMM). services music therapists provide, there is significant need among our country's service men and women for ongoing and additional treatment. According to estimates of the U.S. Department of Veterans Affairs (VA) [7], PTSD afflicts:

- 30 percent of Vietnam veterans

- 12 percent of Gulf War (Desert Storm) veterans

- 11 percent of veterans of the war in Afghanistan

- 20 percent of Iraqi war veterans

The scope and severity of the results of trauma, including PTSD, have been well-documented across disciplines. As a music therapist serving veteran PTSD populations, these needs have been personally observed and echoed by peers and fellow interdisciplinary staff, as well as among our treated veterans. Ongoing efforts to improve services for veterans have been a long-time national focus, yet gaps persist and resolutions can feel slow-moving in spite of these efforts.

As investigation advances toward the development of current best practices to better serve veterans, the military and their families, supported by both the VA and our music therapy professional body $[3,6]$, it is important to also be cognizant of development in trauma psychology and neuroscience to elevate our clinical standard, to formulate and conduct empirical research that fully reflects the quality and impact of services, and to articulate an evidenced-based methodology that is meaningful within an overall clinical treatment plan. With concurrent personal methodology in development, I am striving to return to my foundations of an integrative, trauma-centered music therapy approach to offer renewed perspective into the work with veterans in treatment for PTSD. It is my the intention to utilize the following case review to build upon previously formulated methodology [8] while exploring both the incorporation of current 
traumatology for coping with PTSD and identifying additional needs for growth within music therapy and trauma practice for veterans.

\section{Background}

Acceptance of what is without criticism is both a valuable clinical stance and a classic life principle that helps to open new opportunities for growth and change. Whether regarding the journey of the development of music therapy and trauma practice, the personal journey of the therapist or the journey of each veteran leading to arrival at our clinical door, perhaps Don McClean's words can offer a useful reminder of the potential clinical implication of a non-judgmental and inclusive therapeutic viewpoint:

"You know I've heard about people like me
But I never made the connection.
They walk one road to set them free
And find they've gone the wrong direction.
But there's no need for turning back
Cause all roads lead to where I stand;
And I believe I'll walk them all
No matter what I may have planned."
"Crossroads,"
1971

These words have re-minded me both personally and professionally of the high value of perspective and acceptance in life and in our work. Journeys of life and of work encompass a vast and humbling series of converging paths, experiences and relationships that have the potential to confound as much as inspire. It seems that as we begin and progress through our various courses, our perspective shapes us, directs our thoughts, opinions, beliefs and values. However, these lyrics seem to also be saying that beyond this, and sometimes quite by accident and without our conscious planning, there is the potential to open the soul to the depths of itself, to break us open as never before imagined and know anew that we have the capacity to direct our perspective intentionally; to not be victims of our unconsciousness. To see and accept the beauty of all that this reveals, for me, is the truest purpose, the core and heart of life un-adulterated, that we seek for ourselves and that is our responsibility to not only seek with our clients, but to see as already there beneath the rubble of the habituated mal-adaptations that lead them to treatment.

The context of the case below has been formed by the interplay of standard key treatment factors - roads - in music therapy: namely the client, the therapist, the music, and the system. As a member of this converging network, I am attempting to look with fresh perspective on the process of my music therapy practice with veterans, unfolding my professional treatment lens to date to better understand these treatment factors as a new baseline from which to grow. While the treatment environment can also play a notable role in this interplay, it will not be addressed in this review as it moves beyond the scope of this exploration.

\section{The Therapist}

As with many who enter caregiving professions, my professional journey was fueled by a deep passion to understand the complexities of my inner life, to know the potential of change for myself and to use this to support desired change in others. One of my earliest professional experiences was to coordinate the American Music Therapy Association relief effort post-9/11, "Caring for the Caregiver: A 9-week Training in the Use of Music and Music Therapy in Grief, Loss and Trauma" at Beth Israel Medical Center under the direction of Dr. Joanne Loewy. In this program, I was exposed to the work of Dr. Peter Levine and his body-centered approach to coping with trauma called Somatic Experiencing ${ }^{\circledR}$. This approach resonated with my personal sense of trauma, as I was beginning to understand the traumatic impact of certain childhood experiences and had noticed feeling like I was continuing to embody these experiences in my daily life in destructive ways that had previously been difficult to explain. Somatic Experiencing also resonated with my belief in a complex web of inter-connectedness between body, mind, and spirit, and supported my constructions of integrative medical music therapy.

Somatic Experiencing (SE) is an integrative bodycentered treatment for working with people suffering from traumatic stress [9]. Emphasis is placed on the body's natural neurological and physiological response to traumatic stress, with trauma symptomatology viewed as an incomplete stress activation response that has become fixed in the body. SE treatment works to release this stored activation through a resourced discharge process that follows the body's sensory cues with paced, here-and-now orientation, thereby restoring resiliency by expanding the somatosensory window of tolerance $[10,11]$. Clients learn to re-regulate nervous-system responses to traumatic memories and the emotions attached by identifying and employing balancing internal healing vortices [11].

Insights gleaned from my first introduction to SE led me to pursue additional training and supervision in this treatment approach, ultimately receiving certification as an SE practioner and incorporating SE as an integral component of my music therapy practice. Key features of this approach that inform my work include: 1) felt sense - a conscious embodiment of one's inner landscape, 2) tracking - observing the inner landscape of sensation, feeling, thoughts and images, as well as movement in time/behavior, 3) pendulation between sense-memory experiences that feel life-enhancing/expanding and those that feel frightening/constricting to promote a 
paced and manageable release of traumatic material/discharge of stored energy, 4) resourcing - reconnecting to an inner sense of resiliency and stability, 5) creative self-regulation - a capacity to internally modify the body's state by activating, tolerating, balancing, and releasing energy to restore and maintain equilibrium.

In reference to cultivating a journey of self to Self, I once read that it doesn't matter where you start - body, mind or spirit - as the innately inter-woven quality of these aspects of being will ensure that all are affected. This belief seems to epitomize a 'user-friendly' construct of our inherent and instinctive human capacity for change and has also become a fundamental component of my work through a transpersonal/integrative theoretical foundation. Integral psychotherapy theory offers a relevant platform of inquiry that synthesizes diverse aspects of treatment from a client-centered perspective that is all inclusive [12]; "Integral theory attempts to bring together the most possible numbers of points of view on an issue, with the intention of creating more multifaceted and effective solutions to individual and social problems." [13 p.10] This perspective feeds my detail-oriented mind, my need for thoroughness, and my belief that there are a multitude of "right" points of view, all of which are linked in an organic and masterful web of healing potential. From this theoretical structure, clients are seen through four complimentary and inter-related quadrants of experience direct phenomenological, empirical, collective values and meanings, impersonal observation of the societal system - and across levels of self-identity, lines of development, states of cognition and emotion, and personality orientations [13 p.16]. Integral theory also supports a therapeutic stance of inclusivity that can serve to counter the separateness often perpetuated through habituated silo-ed patterns of thinking and practice.

\section{The Client}

The familiar definition of trauma and the resulting symptomatology that characterizes the nature of PTSD are classified as direct or indirect exposure to death, threatened death, serious injury or sexual violence with intrusion symptoms, persistent avoidance, negative alterations in cognition and mood, and alterations in arousal and reactivity [14]. Symptoms must last more than one month, create distress or impairment, and not be a result of medication, substance use or other illness. PTSD is noted to be one of the most common mental health disorders and has a significant body of research [15]. Veterans coping with PTSD also often present with additional complicating medical and psychiatric diagnoses $[15,16]$. According to Cheryl Gonzales-Nolas, MD, of the James A. Haley VA Hospital in Tampa, FL, veterans seen in VA treatment facilities show a $66 \%$ rate of comorbidity, with issues of Bipolar Disorder and PTSD raising comorbidity rates to $75 \%$ [17]. She further states that the term "dual diagnosis" is now rarely used to describe issues of combined addiction and mental health disorders as most cases involve multiple, co-occurring conditions that may span medical, psychological, social and financial domains.

Beyond a strictly diagnostic description of these clients, common variances in bio-psycho-social parameters consistent with other music therapy treatment populations are important to consider, including age, medications prescribed, cultural origin, socio-economic status, education, familial and employment histories, geographic community identification, religious affiliation, and prior treatment history. Veterans served in a VA PTSD community are also distinguished by their military and trauma histories, potential criminal records (often related to substance abuse history) and past and current suicidality. Understanding a broad client representation is necessary to the evolution of a whole health treatment scope that can target the complex issues faced by veterans recovering from exposure to trauma.

\section{The System}

The system within which we practice may encompass standard methods of treatment as well as structures placed by the institution through which services are received. Phaseoriented treatment has long been the standard of care in trauma renegotiation $[15,18,19]$. Expanded versions have developed since Janet's and Herman's conceptualizations, and have been compiled here with consideration of an integrative, body-centered approach $[8,15,18-21]$ :

- Safety, Stabilization and Symptom Management

- De-conditioning negative patterns, Building selfregulatory capacity

- Processing of traumatic memories, Integrating trauma history into a coherent life narrative

- Re-establishing, re-developing healthy social connections

- Accumulating restorative emotional experiences

- Developing self-care plans, Movement to selfempowerment, Re-engagement in meaningful life experiences

While the processing of a single, event-based trauma may progress relatively smoothly through phrases of treatment, addressing complex and developmental trauma is known to require extended time in the stabilization phase [22]. As such, moving forward into the processing of traumatic memories may be contraindicated for clients with complex PTSD due to vulnerability of decompensation. This level of treatment may also be declined by the client when valuable and satisfactory quality of life measures have been deemed as already met [22]. Additionally, clients commonly move through treatment in a non-linear fashion, cycling in and out of stages, particularly in complex cases, as untangling the web of reactivity involves 
guiding the client to individuate each event as a means to restore the inner timeline and pace processing.

Inclusivity in the evaluation of effective trauma treatment within a system of practice can appear problematic when considering that significantly more approaches in service of trauma recovery have been introduced than have been soundly researched to justify inclusion as evidenced-based practice $[15,22,23]$. Without evidence to back clinical experience, many institutions will be forced, due to liability and need for concrete justification, to deny approval for use or recommendation of otherwise efficacious practices.

To receive recognition within the VA as recommended treatment for PTSD, techniques and approaches must be evidence-based. Empirically-informed trauma treatment currently used as first-line practice within VA programs include prolonged exposure, cognitive processing, eye movement desensitization and reprocessing (EMDR), brief eclectic psychotherapy, narrative exposure therapy and written narrative exposure [24-26]. Approaches recommended with caution due to the need for additional substantiated research include stress inoculation training, present-centered therapy and interpersonal psychotherapy [26]. Recognized as adjunctive therapies, other considered strategies are those backed by trauma neurology research, including yoga, along with t'ai chi and qi gong, and biofeedback, comprising heart-rate variability training and neuro-feedback $[15,18,27]$. Creating cutting edge practice that can qualify as evidence-based requires continued utilization of scientific advancements in related fields as premises for new inquiry as well as for deepened understanding and validity of techniques demonstrated as effective through successful clinical outcomes.

While SE has been recognized as influential in trauma treatment $[15,18,28]$, limitations in the significance of scientific research of SE to date, similar to that in music therapy and trauma, have impeded its recognition as an evidenced-based practice for trauma recovery. While additional research is needed, the first randomized trial in the use of SE for PTSD has shown promising results for decreasing PTSD symptoms and symptoms of depression [29]. Combining SE and music therapy treatment techniques in an integrative mind-body approach may serve to enrich current methods of trauma processing across phases of treatment while avoiding the over-whelming and potentially damaging effects of reliving traumatic memories through intensive narrative exposure [29].

When first assigned to build a music therapy program within our VA communities, I was admittedly naïve as to the impact that the vast federal VA system would have within the campuses I was hired to serve. The common tongue-andcheek statement often shared among VA employees: "You know what they say, if you've seen one VA, you've seen one VA," speaks to the diversity that persists in VAs across the country. As such, it seemed important to first focus on creating a personal standard based on my trauma training and clinical experience rather than seeking to match my program to an existing standard.

Developing programming in the residential communities treating substance abuse and PTSD, and addressing domiciliary rehabilitation, began from scratch due to no prior music therapy services, and emphasis placed on how quickly I could formulate and begin programming within a limited time-frame, allocating one half-day per community per week. This time-frame continues to be one of my primary obstacles to providing the extent of services that I feel would best meet the needs of these veterans and a high standard of care. Strategizing new ways to expand services in these communities without creating a loss of services in others is a consistent challenge. Additionally, attending interdisciplinary rounds has not been prioritized by administration within this schedule, so I have taken initiative in my personal schedule, as I am able, to collaborate with team members during morning reports without compromising clinical time.

Within the available clinical structure, I have carved an opportunity for one group per community per week, and 1-4 individual sessions per week, depending on the time-frame I was assigned and the time that was available within each community's full treatment schedule. In the PTSD community, veterans are regularly rotating in and out of the program and have a 50-day treatment cycle, if not discharged irregularly or having their treatment cycle extended to accommodate transfer needs. This structure creates inconsistency in group composition and potential size. Another factor contributing to a lack of consistency in group attendance is that Art Therapy programming runs concurrently with the Music Therapy group time-frame. As a collaborative measure with the art therapist and to open accessibility of both creative arts programs, veterans are offered to alternate between art and music therapy. Though not ideal, I feel it is preferable at this limited gathering stage than to restrict potential exposure to music therapy as a resource for any veteran who desires it. During program orientations offered upon admission, I also point out options for participating in both programs without having to alternate in and out of the music therapy group.

Beyond the limitations of time and budgetary restrictions making additional music therapists prohibitive at this time, I was given full scope of planning and decision-making in the development of programming, for which I am most grateful. To accommodate rotating enrollment, each group was developed to stand alone and each was designed to address either a specific PTSD symptom or a general emphasis of stability and coping. Often these focuses are intentionally alternated to offer a predictable cycling between familiar uses of music (pre-composed songs) and potentially new and "outof-the-box" techniques that may increase a sense of vulnerability simply by their newness (improvisation, songwriting, music visualization, drumming, vibration, body- 
percussion and tapping, music relaxation, and music and story/myth). Every group is also structurally and musically framed in ritual to enhance feelings of safety, predictability and containment; warm-up uses non-music and musicsupported mindfulness through a breath and body-centered induction, progressively transitioning to both structured and improvisatory music-making as an organized bridging of inner and outer awareness in the here-and-now, and group closure uses a veteran-selected preferred song from a community songbook compiled of peer selections of songs that "always make me (the veteran) feel good."

\section{The Music}

The expansive role of music in life, relationship and treatment is of universal understanding. The impact of music over the spectrum of experience has led to varying degrees of clinical and scientific inquiry across known areas of processing, including perceptual, autonomic, cognitive, emotional, behavioral/motor, hemispheric heterogeneity, and biochemical [30]. Moreover, the use of music in music therapy may be debated as equally expansive depending on the skills, goals, beliefs and preferences of both the therapist and the client. While it is beyond the scope of this article to explore the mysteries and facts known in each of these areas, it is important to remain aware of the full scope of music's role in treatment as an additional perspective in the case to follow and as a reminder of the critical research yet to be pursued to validate what we clinicians have long witnessed of its impact.

For the purposes of this review, it may be helpful to further contextualize the use of music within the music therapy programming designed for the PTSD community in which I work. As often as possible, music choices are made by the veterans involved to better personalize experiences and facilitate associations to the music. This opens the range of music used across genres and eras, though can also restrict the range of music to the familiar song structure most commonly chosen. Efforts to introduce veterans to music outside of typical and traditional listening is done with sensitivity and coupled with psycho-education to support a meaningful rationale and understanding for participating veterans, as well as to invite increased awareness of independent applications post-discharge. It is also wise to prepare veterans and/or inquire as to the familiarity of potential music to be shared, as one veteran's favorite resource song could be another's trigger song.

The value of understanding the impact of music on the brain and body becomes particularly heightened when considering its application in a body-centered, traumainformed music therapy practice. "Musical behaviors activate nearly every region of the brain that has so far been mapped." $[31, p .15]$ While research continues to uncover the vast scope of music's effect on humans and across humanity, investigation of the impact of music from a neurological perspective is still in its youth [32]. Some key links, though this is not an exhaustive representation, illustrate music's capacity to synchronize brain responses across listeners [33], to alter brain structure, to evoke state arousal and improve state regulation $[31,32,34,35]$, to engage neural regulation of the Social Engagement System [32,35], and to engage neurochemical systems of reward, motivation and pleasure [32].

\section{Emilio}

This client case will illustrate an individualized music psychotherapy approach for veterans diagnosed with chronic PTSD. Aspects of this treatment process are referenced through an integrative and multi-modal focus that fuses the body-centered techniques of SE with key principles of music and music therapy in meeting veteran needs and goals. The incorporation of SE techniques will serve as a means of promoting stabilization and developing resiliency by increasing awareness of sensate - felt sense - experience to help resolve fixated physiologic states due to traumatic stress. This case spans clinical contacts over multiple admissions and program transfers, reflecting the common occurrence of substance use/abuse as a means of self-medication that often leads to addiction and relapse on the road to recovery. Repeat admissions and program transfers are also common in trauma recovery as this process often occurs in layers of digestible material spaced over time and veterans typically require stabilization within an acute psychiatric or substance abuse program prior to entering specialized PTSD treatment. Further, the course of music therapy treatment presented in this case helps to illustrate a progressive development of the therapeutic relationship and highlights varied contexts that offer a view of music therapy treatment across a continuum of care. For the purposes of confidentiality, the name of the veteran and select details in this case has been changed.

Emilio is a 30 year old single, never married, heterosexual Latino man, raised in Queens, NY by his mother, who he describes as warm and loving, though always working (noting he was left alone for long periods). Mixed reports of his father include both never knowing him and, alternately, noting that when he was around, they drank heavily and fought. His family also includes one older sister with whom he is estranged. Emilio reports growing up Catholic, though he has not continued formal faith practices, and describes himself as of average intelligence, though he was held back a grade in school (unclear which), received grades of Cs and Ds and was occasionally in trouble at school in his youth. At age 11, he witnessed the collapse of the twin towers on September 11, 2001 from his school room window, identifying this moment as motivating his decision to enlist, which he did at age 17 . There is no history of post-graduate education. 
In addition to 4 years of active service in the Army, Emilio served as a reservist for one year, then later for 3 years. While in combat in Iraq, he experienced multiple traumas that involved explosions, loss of consciousness, multiple firefights, and witnessing the shooting and death of a friend. He remains unemployed since his military service, though maintains financial stability through benefits of his service connection. He is now un-domiciled after being evicted by his mother due to exhibiting unusual behavior, and has a five year history of severe alcohol dependence post-service, with three arrests due to disorderly conduct.

Along with substance abuse, diagnostic issues for Emilio include chronic PTSD, bipolar disorder, alcoholic hepatitis, migraines, cervical spine, post-concussion syndrome due to traumatic brain injury, sinus tachycardia, anxiety and right knee pain. He has a history of multiple psychiatric admissions and residential treatment for substance abuse, PTSD and domiciliary rehabilitation, and was receiving outpatient VA care prior to incidents triggering the recent binging behavior that led to re-admission. Current symptomatology spans all diagnostic clusters, with Emilio expressing experiences of intense irritability, anger, hyper-vigilance, recurrent memories, nightmares, intrusive and racing thoughts, chronic depression, chronic anxiety, loss of sleep, and exaggerated startle response. Other stated and/or observed reactions involve social isolation/withdrawal, avoidance, hyperactivity, hypomania, mood/affect incongruence, grandiosity, pressured speech and emotional numbing. Pharmacology treatment has targeted pain, anxiety, depression, nightmares and insomnia, with strong recommendations for mood stabilization, which Emilio has consistently resisted. No history or current suicidal or homicidal ideation is noted.

Despite this over-whelming array of identified issues, Emilio describes himself as insightful, motivated for change, optimistic that change can occur, moved to re-connect with community, and inspired to meet his full potential. He identifies his mother and sobriety sponsor as important social supports and reports that engagement in creative experiences - music, dance, art, writing - are important resources for helping him to cope with his addiction and PTSD symptoms. Emilio also stresses that he feels safe within this VA treatment environment, and understands that feeling unsafe due to recent experiences in NYC had a triggering effect for him. He recognizes a capacity to utilize treatment based on past experiences, and reports feeling able to manage the demands and potential opportunities that the treatment community is designed to provide.

These descriptions reflect documented professional and client evaluations and statements of report in Emilio's case file. As a medical file, it may be noted that this form of clinical documentation shows a tendency to objectify the client and weighs heavily on diagnosed and perceived areas of difficulty in defining parameters and goals of treatment. While it is an important choice of the therapist whether to read a client's case file before or after the first encounter, it is our responsibility to read it, know it, and be able to communicate knowledgably with fellow professionals on its contents when assuming an active role in the client's individual treatment. Though not always organized succinctly in any one professional's assessment, it seems significant to note that the scope of bio-psycho-social features available through this chart review address all mentioned categories: age, medications prescribed, cultural origin, socio-economic status, education, familial and employment histories, geographic community identification, religious affiliation, prior treatment history (see below), military and trauma histories, criminal records and past and current suicidality.

When Emilio originally presented to music therapy, it was upon his first admission to the Substance Abuse Recovery Treatment Program (SARTP) through the Introduction to Music Therapy group. As a required component of the SARTP program, this is a large group traditionally ranging from 18 28 participants, making in-depth evaluations of each participant unrealistic. Still, Emilio's initial engagement in this group was memorable. He appeared open and enthusiastic, if hypomanic and disorganized at times and with expanded affect, giving the impression of a "live wire," as well as someone who was eager to please and needing attention. $\mathrm{He}$ contributed to group discussion and engaged well in musicmaking opportunities, which included use of familiar song structure for connection and cohesion, lyric analysis for fostering self-reflection, drumming through rhythmic ostinato for evoking feelings of constancy, unity and predictability, echoed lyric improvisation over ostinato for self-expression and validation, and veteran-preferred music for continued personalization in processing. While active in his participation, Emilio showed limited inter-personal awareness in his interactions and needed occasional redirection to counter-balance his dominant energy in the group. Interventions in this introductory group exposure to music therapy are designed to maximize connections to primary life resources through the use of music and the therapeutic community. The primary focus of this first group for Emilio was the role of faith in recovery. Additional resource themes explored during his residency in this community included supportive relationships, nature, stabilization and selfidentity.

After completing the then 28-day SARTP program, Emilio was transferred to the PTSD program, where he engaged well in the first 4 weeks of a then 45 -day treatment cycle. The Music Therapy \& Trauma group offered in this community uses SE principles to emphasize embodied experience while addressing all phrases of trauma treatment as relevant to organic group process and level of stability. The group invites engagement in active and receptive experiences that highlight targeted strategies for the use of music in coping with PTSD symptoms. During his time in this group, structured themes included balance/self-regulation, anger, 
depression, and loss and grief. There were two additional music events offered during this admission for Emilio: African Drumming, inviting a community-based engagement opportunity that emphasized empowerment and commitment to recovery, and The Open Mic Project, which invites all residential veterans, staff, volunteers and family to engage in a live music event through therapeutic performance and community involvement.

Each week during his treatment cycle in the PTSD community, Emilio appeared to become progressively more withdrawn. At the midpoint, he stopped coming to this group. As he also chose not to engage in individual music therapy treatment at this time, I tracked his treatment peripherally. Positive outcomes observed during music therapy groups, such as constructive social engagement, insightful selfexpression and awareness and emotional engagement and regulation, may be likewise deemed as meaningful in the moment, though lacking the capacity for more in-depth individualization and systemic integration.

After completion of and discharge from the PTSD program, Emilio was re-admitted to SARTP within four months, and progressed similarly through this next admission. However, this time he chose to transfer from his completion of substance abuse treatment directly to domiciliary rehabilitation, without admission to the PTSD program. Once admitted to the domiciliary program, engagement in music therapy was limited due to program availability and lack of veteran pursuit for individual follow up. He was discharged irregularly due to relapse.

Emilio's next admission marked his third to this campus within less than three years. As such, I took the initiative to track Emilio on the acute psychiatry unit, where he was first placed. While not professionally assigned to this unit of care, this exception has become a common practice of mine for veterans who have shown a repeated history of relapse and readmission, as well as have demonstrated a strong connection to music as a resource during prior admissions. This initiative is intended to provide, in addition to continuity of care, a personalized outreach to candidly review treatment history and current perceived areas of difficulty, and to strategize on the potential use of individual music therapy treatment as an integrative part of treatment in support of increasing foundational coping resources.

Emilio greeted me with a surprised expression, smiling and hugging me before asking why I'd come. He seemed amazed that I would make the effort to locate and offer time just for him. It was gratifying to see this response from Emilio, both for the potential implications of indicating his assigned value to prior contacts and also for the display of openness and eagerness for renewed contact. Once a private space was identified to invite trust and decrease environmental stimulation, Emilio was engaged openly in a candid review of circumstances leading up to his admission, as well as current commitment to recovery. He was at times provocative and grandiose in describing the extreme nature of his recent binge, near death experience, and believed capacity to detoxify himself, which appeared in the moment more as an attempt to shock me than as a sincere disclosure. With reorientation to documented realities, his affect softened and he spoke with genuineness of his reasons for self-presenting to urgent care, stating, "I don't want to die." "I can't do this anymore." Then, "I'm going to be 30 !" (He turned 30 during the course of this admission.)

With renewed commitment to his recovery, Emilio was oriented to his capacity for survival and to current strengths and resources, which pointed to his faith and creativity. He confided that he dreamed of performing, though this was imbedded with idealism. He also spoke of feeling constricted and suppressed in terms of his self-expression and creative engagement when in treatment. When explored, he demonstrated limited insight into rationale for community structures for the support and benefit of all residents, speaking of constraints placed on where and how loud he could sing on the unit, which had been identified by staff as attentionseeking behavior. After validating his feelings of perceived oppression and injustice, I aimed to reframe his expressions of disconnect and signs of maladaptation by mirroring Emilio's statements and asking if he might consider that another alternative to these thoughts and resulting resistant behavior could exist. He paused, appearing to mentally search for a reason to refute this suggestion before hesitantly agreeing. Now presenting his responses as a matter of choice, I menu-ed his prior scenario alongside additional possibilities of response, emphasizing the potential value of practicing slowing his reactivity in the moment by pausing, breathing, and by bringing focused awareness to his sensate experience in order to restore stabilization and a sense of capacity. In SE terms, this refers to bringing awareness to one's felt sense experience. This awareness functions to connect and attune mind and body experiences in the moment, engaging selfregulation and de-escalating arousal.

Live music was then introduced to increase access to felt sense experience. I suggested recreating a song that spoke to his described feelings of oppression, and was also coupled with themes of faith and fight for freedom - Light One Candle (written by Peter Yarrow) - to facilitate a connection to felt sense through singing, as well as to evoke feelings of validation, perseverance and liberty. Emilio shared that the song was unfamiliar to him, but he became intrigued by the content once lyrics were provided. He was encouraged to help $\mathrm{read} / \mathrm{sing}$ the song to assist me in the music as I had conveniently missed-place my glasses, which wasn't planned but felt like a serendipitous opportunity to invite collaboration and shared intimacy. Having one printed copy of the lyrics also created a need for closer proximity to share the copy.

Entering into the music felt like opening a new door of awareness and being; there was a palpable sense of anticipation that seemed to be a shared undercurrent in this 
moment, not expressed by either Emilio or myself, though felt on a sensory level for me and observed in Emilio as an intention of shared focus. The song's music is embedded with shifts between major and minor tonality, and I used an emotive vocal and instrumental (guitar) dynamic and phrasing to further activate associative physical and mental connections and to stimulate emotional resonance. Emilio leaned his body over the lyrics, showing a continued deepening of focus that added to his quality of investment, while also displaying an increased range of expression through his singing and dynamic variance.

Up to this point, while Emilio and I sat closely side by side, interactive engagement was observed as parallel in nature. I wanted to follow his body language sensitively as a cue of his preferred pacing as well as a tool to bring awareness to authenticity in his contact as well as to deepen trust in this relationship. I felt rewarded for my patience when he soon began to sing in unison with me, "Don't let the light go out! It's lasted for so many years...," making eye-contact in a way that appeared to search for validation and understanding. To dwell in this achieved connection and creative resiliency, the chorus was repeated with final punctuation, energy and vocal inter-play. As the closing music hung in the air, I sustained an intentional pause to create further suspension in the moment. Emilio's affective expression now held an observable sense of vitality and affirmation. "This is me!" he said. "Can I keep these lyrics?" This was an exhilarating moment in the contact. The energy was magnetic, and in addition to reflecting this state with Emilio, I was motivated to consider how to extend this achieved state beyond this immediate moment.

This led me to suggest that Emilio write his own lyrics on this theme of perseverance and personal inspiration for next session. This assignment appeared to excite him as he jumped, held his hands up between us in a 'stop' gesture and exclaimed, "I want to give you something! I'll be right back!" He returned with a personally-colored depiction of the World Trade Center attack of 9-11, which he quickly over-turned saying, "Not that. Look at this side!" On the back of his picture was a list he defined as his goals for professional development. Number one on the list was "Entertainment," which was identified as pursuing music and writing. "I didn't used to be like this," he stated. "I used to be much friendlier in high school," and since then "I've been working to get back to myself." This enthusiasm for self-recreation was mirrored and celebrated, though I also experienced it as grandiose. Therefore, I was moved to not just support his achieved state of self-awareness and aliveness, however notable given his recent near-death experience, but to ground and direct this energy into an intention for treatment. I found myself making a demonstration of taking a deep, audible breath, then drawing attention to his displayed state of excitement as a grounding technique, and asking him to repeat this breath with me so that we could consider using this impetus to jointly set his goals of music therapy treatment. We were then able to collaborate in developing goals of authenticity, self-identity, self-awareness and self-expression, and made a plan for follow up.

Before concluding, I also felt it was important to discuss his drawing on the other side of his list. He described this depiction of 9-11 (unfortunately, there is no consent for publication of the artwork) as his view from his school window on the day of the event. There was youthfulness to his artwork which I now recognize as a characteristic of his hypomanic states that seems to connect to a related level of immaturity and self-absorption as well as vulnerable innocence. He described the event as his motivation for joining the military, as a perceived duty to his country, and conveyed a now familiar tone of injustice this event represented for him.

Unfortunately, conflicting veteran appointments and scheduling delayed follow up with Emilio for a couple of weeks, and our next contact occurred after he transitioned to the SARTP program. In this program, he began to attend the Introduction to Music Therapy group being offered by a music therapy intern. I supervised this group and observed intermittently while meeting with Emilio individually during his unstructured time. To further develop insights gleaned from our initial contact in acute psychiatry, a formal music therapy assessment was conducted using mixed mediums of music and visual art to reflect veteran interests and to broaden tools of expression while supporting increased awareness of Emilio's felt-sense. In addition to goals of assessment, intentions in the contact included exploration of Emilio's optimal and tolerable arousal states, or window-of-tolerance, and continued development of self-regulatory capacity. Incorporated assessment and treatment elements were psycho-education, instrument exploration with improvisation, favorite song identification, musicvisualization, song assignment to stages of stabilization and recovery, and song re-creation.

Stated goals were reviewed with Emilio due to the long intermission since last contact. Psycho-education materials depicting primary skills of stabilization with supportive uses of music were then examined, emphasizing grounding and centering techniques as a means to prepare for the session and to encourage independent daily practice. Techniques reviewed as a means of menu-ing treatment opportunities included breathing exercises, music meditation and relaxation, drumming, body percussion, humming, tapping, instrument skill development, free instrument exploration and song recreation. Emilio was then introduced to music and art materials as a possible palette of expression, and selected the chimes for its soothing quality. He was engaged in creating an improvisatory soundscape of a soothing environment, both as a reflection of his organic stated preference of instrument as well as a means to co-create a desired state of safety and comfort as a self-regulatory reference point. I offered guitar and vocal support using open major $7^{\text {th }}$ chords in a repeated I 
- IV holding pattern in the key of $\mathrm{C}$, then adding the $\mathrm{V}$ chord to the progression for mild development while maintaining musical containment and predictability. After he spontaneously chose to add gentle tapping on a miniature djembe, he rested into a combined fluidity and steady beat in his play. This appeared to indicate a sense of stability and grounded-ness, as well as a readiness for forward development, so he was then invited to close his eyes and explore any surfacing imagery that the music evoked. I chose to keep a relatively consistent ostinato in the music, adding subtle vocal improvisation for melodic development to sustain interest while building an experience of the music as a connection to resource in the body. This was continued for a couple of minutes before mild restlessness was observed, after which Emilio was prompted to use the art materials provided to illustrate his imagery and continue to channel his focus. When completed, he described the imagery as representing openness to new ideas and feeling inspired with uplifting energy that radiated inside and out (seen in concentric circles). He noted the source of the energy as the head (strongest) and then the heart (pure, bright white), and spoke of a connection to a strength in the "un-see-able" (warm yellow between). Emilio was asked to notice his felt sense of this imagery, though this was difficult for him to articulate. With invitational language used to promote tolerance, curiosity and support in this new exploration, he then shared, "It felt good. Inspired," indicating a potential disconnect from his sensate experience. Completing review of his art, attention was lastly drawn to a deep red circle that was connected yet separate from the others, appearing to point to a disconnected and injured part of the self. Emilio also showed difficulty putting words to this image, and was offered reassurance with psycho-education to facilitate a normalization of this response. He was then invited to remain curious, should a thought or feeling about this surface as we moved forward.

Once processing of this improvisation and visualization were complete, the session focus returned to stabilization to support de-escalation of potentially activated traumatic material, stored as arousal in the nervous system (NS) (trauma vortex - sympathetic NS), and re-engagement of selfregulation and resiliency (healing vortex - parasympathetic NS). From a body-centered, SE stand-point, the trauma vortex represents a break in the natural boundaries of the self that results in a pooled and ever-spinning state of heightened arousal, while the healing vortex refers to one's innate center of resiliency $[9,11]$. Working the periphery of these vortices through paced pendulation within the client's window of tolerance is a primary feature of the SE approach to trauma renegotiation, building a sensory tolerance to remain present as manageable pieces of the trauma, stored as somatic experience, are allowed to surface, process and integrate through consistent re-sourcing. Returning Emilio to predictable song structure seemed a logical means to help organize his NS and thus integrate surfaced activation.
As a tool to help Emilio bring awareness to varied states of experience across a continuum of stabilization, I developed a visual and musical reference called a 'Song Palette,' to appeal to Emilio's diverse creative strengths. My intention was to use the illustration of an arc to represent a progression of increasing capacity, using familiar and/or preferred songs to match associated feelings along the trajectory from least to most stable, with a clear turning point represented at the top of the arc. I planned an example of songs across the trajectory to promote conceptualization, with the aim of having Emilio personalize the song choices over time.

Emilio was oriented to the song palette example, which included 8 songs arranged from least (1) to most stable (8): 1.Behind Blue Eyes (composer: Peter Townshend), 2.Numb (composer: Linkin Park - Chester Bennington, Mike Shinoda, Joe Hahn, Dave Farrell, Brad Delson, and Rob Bourdon), 3.Your Own Worst Enemy (composer: Bruce Springsteen), 4.Tell Your Heart to Beat Again (composer: Matthew West and Bernie Herms), 5.Unwritten (composer: Natasha Bedingfield and Danielle Brisebois), 6.The Climb (composer: Jessi Alexander and Jon Mabe), 7.Rise Up (composer: (Cassandra Batie and Jenn Decilveo), 8.The Greatest (composer: Sia Furler, Greg Kurstin and Kendrick Duckworth). Song examples were menu-ed and explored for familiarity, and Emilio was asked to choose his preference for the day, as per which song(s) he was most resonating with. He conveyed having a relationship with both Numb and The Greatest, referencing feelings of oppression and desires to rediscover a fuller sense of self. Therefore, these songs were offered in succession to honor each as meaningful in his personal journey while inviting another small pendulation of energy from the edge of the trauma vortex (Numb) to the healing vortex (The Greatest). The songs were re-created to replicate their original known versions acoustically with voice and guitar, and a streamlined use of voice and guitar was intended to transfer emphasis to the lyrical content of the music in titrating processing of mind and body. Emilio declined an additional instrument, choosing to sing along. This experiential shift to side by side singing felt reminiscent of our last contact. Though the moment seemed less intimate in the bright and expansive room we were now in, moving to this positioning appeared to invite increased interpersonal connection and capacity for co-regulation. Emilio's singing showed increased vocal strength from the previous session. He appeared relaxed as he moved through each selection, displaying emotional fluidity and an affect that was congruent with song meaning while maintaining a sense of composure. I was unclear if this composure was rooted in regulation or was reflecting potential fatigue, so I sang in unison with him, matching his dynamics in support of his expression without imposing additional energy. To err on the side of caution, I chose to flow from one song to the next, as a medley, to maintain a musical focus on the movement to resiliency depicted in the second song. After the music, Emilio repeated 
his affinity for these choices and was acknowledged and praised for observed growing awareness, resiliency and strength. Further processing was avoided both due to preparation for closing the session and my desire to maintain the apparent state regulation achieved. The session experiences were then reviewed to emphasize accomplishments, and a favorite song was identified and discussed for exploration in the next contact.

Emilio initially struggled to identify a favorite song, and then named Billy Jean (composer: Michael Jackson). He recalled listening to this song in his youth, brightening as he remembered learning Michael Jackson's choreography and dancing to it in school, as well as sharing the attached emotional memories of joy in freedom of expression felt at that time and in connection to the song. To promote the reestablishment of healthy social connections and to foster a restorative emotional experience, Emilio was invited to perform this song in the upcoming Open Mic Project event, scheduled for the following week. Again, he showed surprise at the possibility of such an opportunity. However, this time his surprise appeared coupled with uncertainty and trepidation. I reminded him of the impact of his recalled memory of youth and his stated desire to reconnect to this lost sense of self. He was also reassured that participating in the event was completely his choice, and he decided he would think about it.

When encountered the following week, Emilio had been transferred to the PTSD residential treatment community and unfortunately the time for contact was limited. He shared that he was still unsure about his decision and was told that his music had been prepared for him, should he resolve to participate. Finally, with the Open Mic Event program underway, Emilio was once more offered an opportunity join and agreed with modest encouragement from peers and treatment staff. The Open Mic Project is a bi-annual program that replicates a traditional community Open Mic format by allowing performers (veterans, staff, family, friends and volunteers) to spontaneously participate on a first-come, firstserved basis. While describing feeling nervous, Emilio also appeared eager for this opportunity by coming prepared with a costume, including a hat and sparkling glove, and turning down lights for dramatic effect. On his cue, I was joined by fellow staff and volunteers in providing musical accompaniment as he sang and danced with great energy, dynamic and accuracy of dance steps. He received a rousing ovation in response and was encouraged to pause in acknowledgment and hopeful acceptance of this feedback, though he appeared awkward in this gesture. After the program, when asked his response to the experience, he again showed difficulty with his ability to accept the praise offered, and was supported with reframing and encouragement. In hind-sight, I regret not structuring his performance with additional opportunities before and after the event to help Emilio better ground and prepare for, as well as contain and process surfaced energy. This feels an important take-away with regard to therapeutic performance opportunities.

Due to inclement weather, the next week's individual session was unavoidably cancelled. In the interim period between contacts, it was learned that Emilio was once more struggling to remain engaged and focused in his PTSD treatment. During the following session, he shared feeling depressed and detached, and recognized these feelings had begun to develop before leaving SARTP. Though feeling "lost," he accepted support in continuing to build new strategies for coping. I chose to move directly into the music to facilitate re-stabilization through multi-sensory, felt sense engagement and emotional entrainment. Emilio was directed to focus on his sensory experience and asked to select an instrument to help deepen connection to this in the moment. He chose a small hand-held drum, which he played quietly and expressively to a collaboratively chosen song from the stabilization Song Palette that was perceived to match his presenting state - Your Own Worst Enemy. This mutual song choice appeared to indicate a resonance in this relationship and awareness for Emilio that on some level he understood he had a role in the development of his current state. I began the music slowly and softly, feeling the fragility of the moment and wanting to delicately coax forward his expressions as ownership and tolerance of this state. Once I observed his commitment to the experience through sustained drumming, I began to build the music's dynamics, contouring poignant lyrics to further draw him forward in resonance with me and support connection with his inner life and attached sensory self. Highlighted lyrics included "The condition you're in, now you just can't get out of this skin," and "The times, they got too clear, so you removed all the mirrors." At the music's midpoint, he was encouraged to set down the drum and begin to free-write in response to the content and feelings evoked by the song. During this time, I repeated a non-verbal phrase in the music that replicated emotional, moaning release through a descending stepwise melody on "Ah, ah, ah...," to continue to evoke his emotional resonance. Then, while maintaining the song structure, I asked him to share his writing how and when he felt comfortable to do so. He began to sing with a quiet strength that felt startling. His improvised melody matched the structure of the music and conveyed an emotive openness and authenticity. His lyrics included themes of freedom, release and inner strength. Vocal energy displayed was supported musically, and increased in volume as his singing progressed. When he finished, his affect had brightened, posture straightened, and he exclaimed, "Yeah! Okay!" His behavior seemed to show that he had surprised himself in this experience and connected to a place of inner integrity, punching his fist in the air. In closing, he was reminded of the importance of daily practice techniques to reenforce his connection to self/voice. When additional song preferences were explored, Emilio was now enthusiastic and numerous in his requests. Later that evening, I returned with a 
player and CDs matching his requests (including Michael Jackson), so that he could begin to independently cultivate this connection to self during his personal time. He responded, "You don't know what this means to me!"

The following week, it was learned that Emilio initiated an altercation with a peer during an Alcoholics Anonymous meeting. His behavior was described as provocative, though he defended that it was not intended to be. The interaction had escalated and required staff intervention to ensure safety. When questioned by staff, he stated feeling provoked and showed limited insight into his role in the incident. Due to the community's policy to maintain safety in this treatment environment, the treatment team decided that Emilio was to be irregularly discharged, and arrangements were made to coordinate his transfer to an alternate VA treatment facility.

When I arrived next on the PTSD unit, I had been informed that he had already been discharged and was surprise to encounter him spontaneously on the unit as he presented to return his loaned materials. He accepted a brief closing contact with observable reluctance, and was escorted to a small meditation room for privacy. With encouragement, he shared his response to the altercation and resulting discharge plan, maintaining feelings of injustice and uncertainty of the reasoning behind this outcome. He also expressed sadness to be leaving and reiterated genuine investment in his recovery. So as not to challenge negative mindsets at this time of closure, as well as to invite connection to capacity, Emilio was offered empathy and redirected to reflect on outcomes of prior admissions with respect to the additional efforts made in his recent enrollment. Emphasis was placed on willingness for self-exploration and development of new coping strategies through individual music therapy processing during this admission. Emilio was reminded of expanded creative resources now available to him and was encouraged to continue to practice his new skills, as well as to reach out to the new facility's music therapist for assistance. He shared that he had saved the lyric sheet for "Light One Candle" that was offered while in acute psychiatry, expressing continued support and inspiration found in the song's message. He was tearful at times throughout this contact, and articulated continued commitment to recovery, expressing thanks for support received. I felt resonant resistance to terminate this treatment process, and a sense of loss to be unable to further our developing relationship. Reflecting back, I recognize that I have no right of ownership over Emilio's journey and must continue to practice my own acceptance of what is to open to a greater potential for the future. In this effort, Don McClean's lyrics resurface and offer me support: "There's no need for turning back, 'cause all roads lead to where I stand. And I believe I'll walk them all, no matter what I may have planned."

\section{Discussion}

This case illustration was presented to prompt increasing reflection on the converging lines, levels, states and styles of being that are woven between therapist, client, music and system to create each unique phenomenon of experience in music therapy and trauma treatment. As with a composition of music or work of art, the eye of the creative master has intimate and intricate awareness of each feature, reasoned and intuited, as well as a vision of the whole. Trauma manifests as a fragmentation of self, the pieces of which often become distorted beyond recognition for its host. As trauma therapists, we become weavers of these fragments, ever aware of the hidden beauty of the whole self that is trying to find its way to back.

Emilio conveyed well the manifested fragmentation resulting from his life journey to date. Creative music therapy experiences appeared to help restore connections to healthy and healing aspects of being during his time in treatment, while increased exposure to and integration of felt sense experience, particularly in increased frequency of contact and progressively structured experiences, may have offered important opportunities for deepened stabilization, desensitization and paced renegotiation of stored somatization of traumatic experiences.

Systemic challenges notwithstanding, objective and subjective evaluation of potentially successful and failed aspects of music therapy phenomena is necessary to assess gaps in treatment, both micro- and macro-spectively, to help minimize ongoing resurgences of incapacitating trauma symptomatology. Viewing treatment through an integrative psychotherapy lens may offer an advanced scope of evaluation to improve understanding in needed avenues of empirical study, teasing apart values of each element of practice in reconstructing evidence-based protocols. Investment in this advancement from governmental and professional bodies creates an excited anticipation of the potential ahead for compiling and furthering scientific findings from research on the impact of music on mind, body, brain and soul with current outcomes for body-centered trauma treatment and the bio-neuro-chemical life of trauma.

\section{References}

1. Rourke MA. Music and the wounded of WWII. J Music Ther, 1996: 33(3): 189-207.

2. U.S. War Department. Technical bulletin 187: Music in reconditioning the American Service Forces convalescent and general hospitals. Report of the U.S. War Department; 1945; Washington, DC.

3. Music therapy and military populations. American Music Therapy Association Website.

http://www.musictherapy.org/assets/1/7/MusicTherapyMilitaryPops_2 014.pdf. Accessed February 8, 2018. 
4. Bensimon M, Amir D and Wolf Y. A pendulum between trauma and life: Group music therapy with post-traumatized soldiers. Arts Psychother 2012: 39(4): 223-233.

5. Davis MT, Mulvaney-Day N, Larson MJ, Hoover R, Mauch D. Complementary and alternative medicine among veterans and military personnel: A synthesis of population surveys. Med Care. 2014 Dec (12 Suppl 5): S83-90.

6. Americans for the Arts. Arts, health and wellness across the military continuum. Report of the Americans for the Arts; 2013; Washington, DC.

7. PTSD: National Center for PTSD resources page. U.S. Department of Veteran Affairs Website. Available at https://www.ptsd.va.gov/public/ptsd-overview/basics/how-common-isptsd.asp. Accessed March 31, 2018.

8. Stewart, Kristen. PATTERNS - A model for evaluating trauma in NICU music therapy: Part 1 - Theory and design. Music and Medicine. 2009: 1(1): 29-40.

9. Levine, PA. Waking the tiger: Healing trauma. Berkeley: North Atlantic Books; 1997.

10. Levine, PA. In an unspoken voice: How the body releases trauma and restores goodness. Berkeley: North Atlantic Books; 2010.

11. Poole-Heller D, Levine PA. Somatic-Experiencing: Beginning level curriculum. Unpublished manuscript; 2002.

12. Wilber K. A brief history of everything. Boston : Shambhala; 1996.

13. Forman $\mathrm{MD}$. Integral theory and the principles of integral psychotherapy. In Forman MD, ed. A guide to integral psychotherapy: Complexity, integration, and spirituality in practice. Albany: SUNY Press; 2010: 9-34.

14. American Psychiatric Association. Diagnostic and statistical manual of mental disorders. 5th edition. Arlington: Author; 2013.

15. van der Kolk, Bessel A. The assessment and treatment of complex PTSD. In Yehuda R, ed. Traumatic Stress. Washington, DC: American Psychiatric Press: 2001.

16. Britvic D, Antiĉeviĉ V, Kaliterna M, Lušić, et al. Comorbidities with posttraumatic stress disorder (PTSD) among combat veterans: 15 years postwar analysis. International Journal of Clinical and Health Psychology. 2015; 15: 81-92.

17. Gonzales-Nolas, Cheryl. Dual Diagnosis [Video]. U.S. Department of Veteran Affairs. Available at https://www.va.gov/.../docs/.../GPDTrn_Dual_Diagnosis_Presentation_ 3-13-14.ppts. Published February 26, 2014. Accessed March 31, 2018.

18. Fatter, Daphne. Utilizing phase-oriented treatment and adjunctive interventions to regulate arousal in trauma treatment home study [Webinar] Clarksville : TZK Seminars, 2018.

19. Herman, Judith. Trauma and recovery. New York: Basic Books; 1992.

20. van der Hart O, Brown P, van der Kolk, BA. Pierre Janet's treatment of posttraumatic stress. J Trauma Stress. 1989: 2(4): 379-395.

21. van der Kolk BA, McFarlane AC, van der Hart O. A general approach to treatment of posttraumatic stress disorder. In van der Kolk BA, McFarlane AC, Weisaeth L, eds. Traumatic Stress: The effects of overwhelming experience on mind, body, and society. New York: Guilford Press; 1996: 417-440.

22. Fatter, Daphne. Integrating traumatic memories: Conceptualization and clinical considerations in evidence-based approaches to trauma processing home study [Webinar] Clarksville : TZK Seminars, 2018.

23. Psychotherapy.net. Evidence-based treatment planning for posttraumatic stress disorder (for counselors)[Webinar]. U.S. Department of Veterans Affairs - Talent Management System. Available at https://www.tms.va.gov/learning/user/common/viewItemDetails.do?co mponentTypeID=NFED\&componentID $=14380$ \& revisionDate $=148190$ 7600000\&fromHistory=true\&menuGroup=Learning\&menuItem $=$ Lear
ning\%20History. Published December 16, 2016. Accessed March 23, 2018.]

24. U.S. Department of Veteran Affairs. Evidence-based treatment planning for PTSD [Webinar]. U.S. Department of Veterans Affairs - Talent Management System. Available at

https://www.tms.va.gov/learning/user/common/viewItemDetails.do?co mponent TypeID $=$ NFED\&component $I D=14380 \&$ revisionDate $=148190$ 7600000\&fromHistory $=$ true\&menuGroup $=$ Learning\&menuItem $=$ Lear ning\%20History., 2016. Published December 16, 2016. Accessed March 23, 2018.

25. Schnurr, PP. Treatments for PTSD: Understanding the evidence. PTSD Research Quarterly. 2008 Summer; 19(3): 1-3. Available at https://www.ptsd.va.gov/professional/newsletters/researchquarterly/V19N3.pdf. Accessed March 23, 2018.

26. U.S. Department of Veterans Affairs and Department of Defense. VA/DOD Clinical practice guideline for the management of posttraumatic stress disorder and acute stress disorder: Clinical summary. Report of the U.S. Department of Veterans Affairs and Department of Defense; 2017; Washington, DC.

27. Foa EB, Keane TM, Friedman MJ, Cohen JA. Effective treatments for PTSD: Practice guidelines from the international society for traumatic stress studies. $2^{\text {nd }}$ ed. New York: Guilford Press; 2010.

28. Bosco F. Translating somatic experiencing and gestalt therapy for trauma resolution into music therapy practice with adults. In Stewart, Kristen, ed. Music therapy \& trauma: Bridging theory and clinical practice. New York: Satchnote Press; 2010: 58-73.

29. Brom D, Stokar Y, Lawi C, et al. Somatic experiencing for posttraumatic stress disorder: A randomized controlled outcome study. J Trauma Stress. 2017 Jun; 30(3): 304-312.

30. Nizamie SH, Tikka SK. Psychiatry and music. Indian J Psychiatry. 2014 Apr; 56(2): 128-140.

31. Levitin, Daniel J. Neural correlates of musical behaviors: A brief overview. Music Ther Persp. 2013; 31(1): 15-24.

32. Chanda ML, Levitin DJ. The neurochemistry of music. Trends in Cogn Sci. 2013; 17(4): 179-193.

33. Abrams DA, Ryali $\mathrm{S}$, Chen $\mathrm{T}$, et al. Inter-subject synchronization of brain responses during natural music listening. Eur J Neurosci. 2013 May; 37(9): 1458-1469.

34. Dillman Carpentier FR, Potter RF. Effects of music and physiologic arousal: Explorations into tempo and genre. J Media Psych. 2007; 10(3): 339-363.

35. Porges, Stephen W. Music therapy and trauma: Insights from the polyvagal theory. In Stewart K, ed. Music therapy \& trauma: Bridging theory and clinical practice. New York: Satchnote Press; 2010: 3-15.

\section{Biographical Statements}

Kristen Stewart, MA, LCAT, MT-BC, holds two post-graduate certifications in trauma treatment, has worked to develop a new model of treatment for trauma prevention in preterm infants (PATTERNS), and has evolved her music therapy practice through her work in medical, educational, mental health and residential contexts. She is currently working with veterans in the VA - Hudson Valley Healthcare System. 